\title{
Do Hydrogen Bonds Influence Excitonic Splittings?
}

\author{
Franziska A. Balmer $\$$, Philipp Ottiger, and Samuel Leutwyler* \\ §SCS-Metrohm Award for best oral presentation in Physical Chemistry
}

\begin{abstract}
The excitonic splitting and vibronic quenching of the inversion-symmetric homodimers of benzonitrile, $(\mathrm{BNN})_{2}$, and meta-cyanophenol, $(\mathrm{mCP})_{2}$, are investigated by two-color resonant two-photon ionization spectroscopy. These systems have very different hydrogen bond strengths: the $\mathrm{OH} \cdots \mathrm{N} \equiv \mathrm{C}$ bonds in $(\mathrm{mCP})_{2}$ are $\sim 10$ times stronger than the $\mathrm{CH} \cdots \mathrm{N} \equiv \mathrm{C}$ hydrogen bonds in $(\mathrm{BN})_{2}$. In $(\mathrm{BN})_{2}$ the $S_{0}\left({ }^{1} A_{g}\right) \rightarrow S_{1}\left({ }^{1} A_{g}\right)$ transition is electric-dipole forbidden, while the $\left.S_{0}\left({ }^{1} A_{g}\right) \rightarrow S_{2}{ }^{1} B_{u}\right)$ transition is allowed. The opposite holds for $(\mathrm{mCP})_{2}$ due to the different transition dipole moment vector alignment. The $S_{0} \rightarrow S_{1} / S_{2}$ spectra of the dimers are compared and their excitonic splittings and vibronic quenchings are investigated by measuring the ${ }^{13} \mathrm{C}$-substituted heterodimer isotopomers, for which the centrosymmetry is broken and both transitions are allowed. The excitonic splittings are determined as $\Delta_{\text {exc }}=2.1 \mathrm{~cm}^{-1}$ for $(\mathrm{BN})_{2}$ and $\Delta_{\text {exc }}=7.3 \mathrm{~cm}^{-1}$ for $(\mathrm{mCP})_{2}$. The latter exhibits a much stronger vibronic quenching, as the purely electronic splitting resulting from ab initio calculations is determined to be $\Delta_{\text {calc }}=179 \mathrm{~cm}^{-1}$, while in $(\mathrm{BN})_{2}$ the calculated splitting is $\Delta_{\text {calc }}=10 \mathrm{~cm}^{-1}$. The monomer site-shifts upon dimerization and comparing certain vibrations that deform the hydrogen bonds confirm that the $\mathrm{OH} \cdots \mathrm{N} \equiv \mathrm{C}$ hydrogen bond is much stronger than the $\mathrm{CH} \cdots \mathrm{N} \equiv \mathrm{C}$ bond. We show that the $\mathrm{H}$-bonds have large effects on the spectral shifts, but little or no influence on the excitonic splitting.
\end{abstract}

Keywords: Benzonitrile $\cdot$ meta-Cyanophenol $\cdot$ Excitonic splitting $\cdot$ Hydrogen bonds $\cdot$ Vibronic coupling

\section{Introduction}

Hydrogen-bonded molecular dimers are model systems to investigate excitonic interactions, which are of great importance in many photochemical and biological systems such as conjugated polymers, molecular crystals and photosynthetic lightharvesting complexes. ${ }^{[1]}$ Such dimers of benzene derivatives are small enough to allow detailed experimental, theoretical and computational investigations and are at the same time representative of larger systems. Our group has been studying the excitonic $S_{0} \rightarrow S_{1} / S_{2}$ splittings in rigid, doubly hydrogen-bonded dimers such as (2-pyridone), (2-aminopyridine), (benzoic acid), (benzonitrile $)_{2}$ and (ortho-cyanophenol) ${ }_{2}{ }^{[2-6]}$

${ }^{*}$ Correspondence: Prof. Dr. S. Leutwyler

E-mail: leutwyler@dcb.unibe.ch

Universität Bern

Departement für Chemie und Biochemie

Freiestrasse 3

$\mathrm{CH}-3012$ Bern
They are centrosymmetric, meaning that the electronically excited $S_{1}$ (and higher $S_{n}$ ) states of monomers $A$ and $B$ are degenerate and excitonically coupled. ${ }^{[2-8]}$ Upon dimerization the transition dipole moment (TDM) vectors of the monomers combine in a parallel and antiparallel manner, giving rise to an antisymmetric $\left(\mathrm{A}_{\mathrm{u}}, \mathrm{B}_{\mathrm{u}}\right)$ and a symmetric $\left(\mathrm{A}_{\mathrm{g}}\right)$ excited state combination. [9] Thus, either the $S_{0} \rightarrow S_{1}$ or the $S_{0} \rightarrow S_{2}$ transition is electric-dipole $(\mathrm{g} \leftrightarrow \mathrm{u})$ allowed, while the other is electric-dipole $(\mathrm{g} \leftrightarrow \mathrm{g})$ forbidden. The inversion symmetry can be broken by isotopic substitution of even a single atom, e.g. ${ }^{12} \mathrm{C} /{ }^{13} \mathrm{C}$ or $\mathrm{H} / \mathrm{D}$, rendering the forbidden transition slightly allowed.[2-6] This enables us to experimentally determine the $S_{1} / S_{2}$ splitting, denoted $\Delta_{\text {obs }}$.

We have previously shown that the displacements along the intra- and intermolecular vibrational coordinates $Q_{i}$ that occur upon electronic excitation and which are quantified by the Huang-Rhys factors $S$, have a major impact on the magnitude of the experimental splittings. ${ }^{[3,6]}$ The observed splittings are up to a factor 40 smaller than the purely electronic (Davydov) exciton splittings $\Delta_{\text {calc }}$ that are predicted by high-level $a b$ initio calculations. This so-called 'vibronic quenching' of the excitonic splitting $\Delta_{\text {calc }}$ can be obtained ${ }^{[3-10]}$ by taking the Huang-Rhys factors $S_{i}$ into account within Förster's perturbation theory approach $^{[7]}$ or the Fulton-Gouterman model. ${ }^{[11,12]}$ Applying the resulting quenching factor $\Gamma$ to the calculated exciton splitting $\Delta_{\text {calc }}$ results in vibronic splittings $\Delta_{\text {vibron }}$ that are very close to the experimentally observed $S_{1} / S_{2}$ splittings.

$$
\Delta_{\text {vibron }}=\Gamma \cdot \Delta_{\text {calc }}
$$

The strength of hydrogen bonds is dependent on the atoms involved and on structural aspects. The shift in absorption energy of the dimer relative to the monomer allows us to draw conclusions on the stabilization gained upon dimerization. Further, vibrations deforming the hydrogen bond also give us information on the $\mathrm{H}$-bond strength. Here, we investigate if hydrogen bond strength has a direct influence on the excitonic splitting and vibronic coupling on the examples of the benzonitrile dimer, $(\mathrm{BN})$, with two $\mathrm{CH} \cdots \mathrm{N} \equiv \mathrm{C}$ hydrogen bonds, and the meta-cyanophenol dimer, $(\mathrm{mCP})_{2}$, with two $\mathrm{OH} \cdots \mathrm{N} \equiv \mathrm{C}$ hydrogen bonds.

\section{Structural Aspects}

The ground-state structures of $(\mathrm{BN})_{2}$ and $(\mathrm{mCP})$, were optimized by the spincomponent-scaled variant of the approxi- 
mate second-order coupled-cluster (SCSCC2) method using the aug-cc-pVTZ basis set and are shown in Fig. 1. (BN), is a planar, $C_{2 h}$-symmetric dimer, ${ }^{[13,14]}$ while $(\mathrm{mCP})_{2}$ is $C_{i}$-symmetric, with the monomers slightly out of plane..$^{[9,15]}$

The calculated intermonomer distance between the centers of mass in $(\mathrm{mCP})$, is $\mathrm{R}_{\mathrm{AB}}=5.34 \AA$ with an $\mathrm{H} \cdots \mathrm{N}$ distance of 2.04 $\AA$; the center-of-mass distance in $(\mathrm{BN})_{2}$ is $\mathrm{R}_{\mathrm{AB}}=6.46 \AA$ and the $\mathrm{H} \cdots \mathrm{N}$ distance is $2.36 \AA$. The presence of the $\mathrm{OH}$ group in $(\mathrm{mCP})_{2}$ changes the transition dipole moment (TDM) orientation: In $(\mathrm{BN})_{2}$ the angle of the monomer TDM relative to the $\vec{R}_{A B}$ vector is $\theta=63^{\circ}$, which is larger than the 'magic angle' for which the magnitude of the dipole-dipole interaction goes to zero, i.e. $\arccos (1 / \sqrt{3})=54.7^{\circ}$.

Thus, the TDMs are oriented parallel/ antiparallel, the $S_{0} \rightarrow S_{1}$ transition is forbidden and the $S_{0} \rightarrow S_{2}$ transition is allowed. For $(\mathrm{mCP})_{2}$, the angle is $\theta=11^{\circ}$, which is smaller than the magic angle. In this case, the $S_{0} \rightarrow S_{1}$ transition is allowed and the $S_{0} \rightarrow S_{2}$ transition is forbidden. ${ }^{[9]}$

\section{Two-Color Resonant Two-Photon Ionization Spectra}

The dimers were produced and cooled in pulsed supersonic jet expansions. BN was heated to $75{ }^{\circ} \mathrm{C}$ in an external vaporizer and seeded into the neon carrier gas (backing pressure 1.2 bar), while $\mathrm{mCP}$ was heated inside the pulsed nozzle to $80{ }^{\circ} \mathrm{C}$. Two-color resonant two-photon ionization (2C-R2PI) spectra were recorded by overlapping the excitation and ionization lasers in the source of a time-of-flight mass spectrometer. The spectra are shown in Fig. 2 with both electronic origins set to zero for comparison. The $0_{0}^{0}$ transition energies are indicated next to the corresponding band, namely $36420 \mathrm{~cm}^{-1}$ for $(\mathrm{BN})_{2}$ and 33255 $\mathrm{cm}^{-1}$ for $(\mathrm{mCP})_{2}{ }^{[9,13]}$ These energies correspond to spectral shifts of $\delta \mathrm{v}=-92 \mathrm{~cm}^{-1}$ for $(\mathrm{BN})_{2}$ and $\delta \mathrm{v}=-1094 \mathrm{~cm}^{-1}$ for $(\mathrm{mCP})_{2}$ relative to the respective monomer $S_{0} \rightarrow S_{1}$ transition energies. The much larger shift for $(\mathrm{mCP})_{2}$ indicates a stronger hydrogen bond of $(\mathrm{mCP})_{2}$ relative to that of $(\mathrm{BN})_{2}$. This will be discussed in Section 5 .

The intramolecular normal modes are denoted according to the Wilson notation, ${ }^{[16]}$ while for the intermolecular normal modes we employ the nomenclature introduced in ref. [17]. The $(\mathrm{BN})_{2}$ spectrum is dominated by intramolecular vibronic bands. Only one intermolecular vibration is observed, the shear vibration $\chi_{0}^{1}$; otherwise the spectrum is very similar to that of BN. ${ }^{[13]}$ By contrast, the spectrum of $(\mathrm{mCP})_{2}$ shows many excitations of intermolecular vibrations $(\delta, \beta, \theta, \sigma, \chi)$ as well as several intramolecular vibrations..$^{[9,15]}$

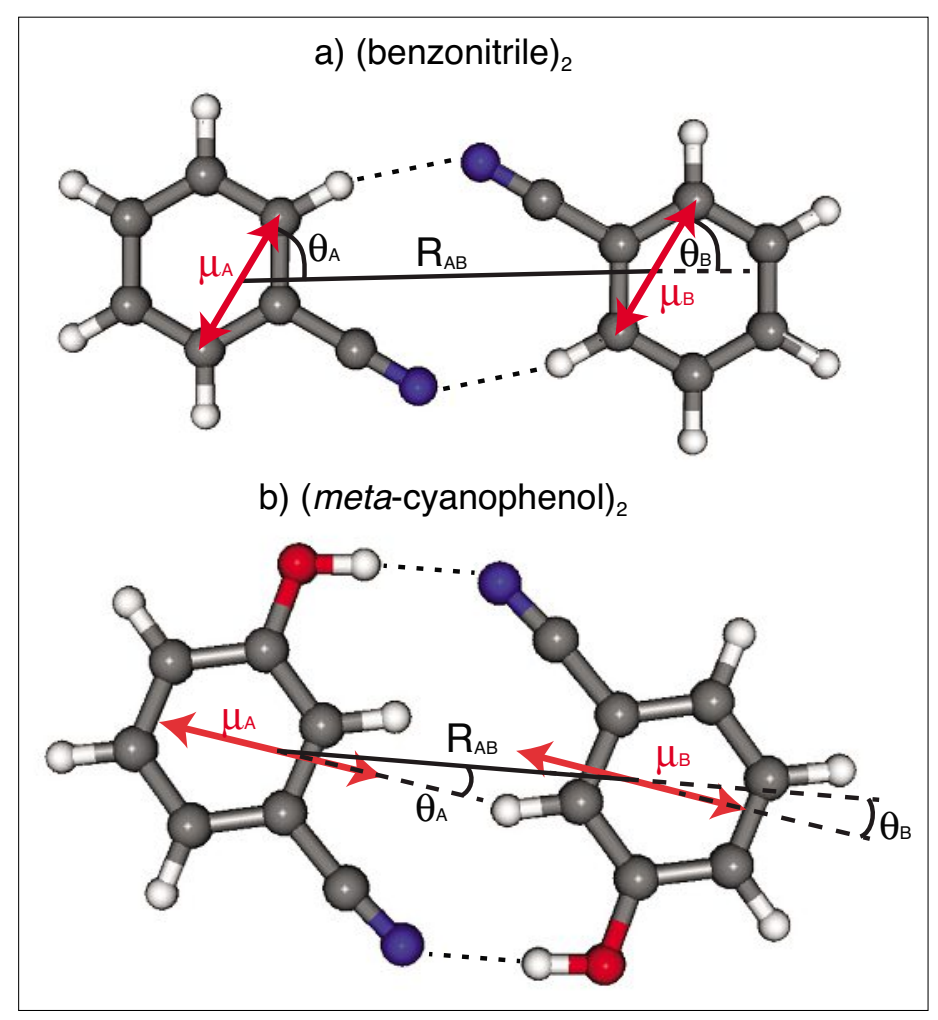

Fig. 1.

a) (Benzonitrile) $)_{2}$ and b) (m-cyanophenol) with indicated monomer transition dipole moments $(\mu)$, intermonomer distance $\mathrm{R}_{\mathrm{AB}}$ and TDM alignment angle $\theta$.

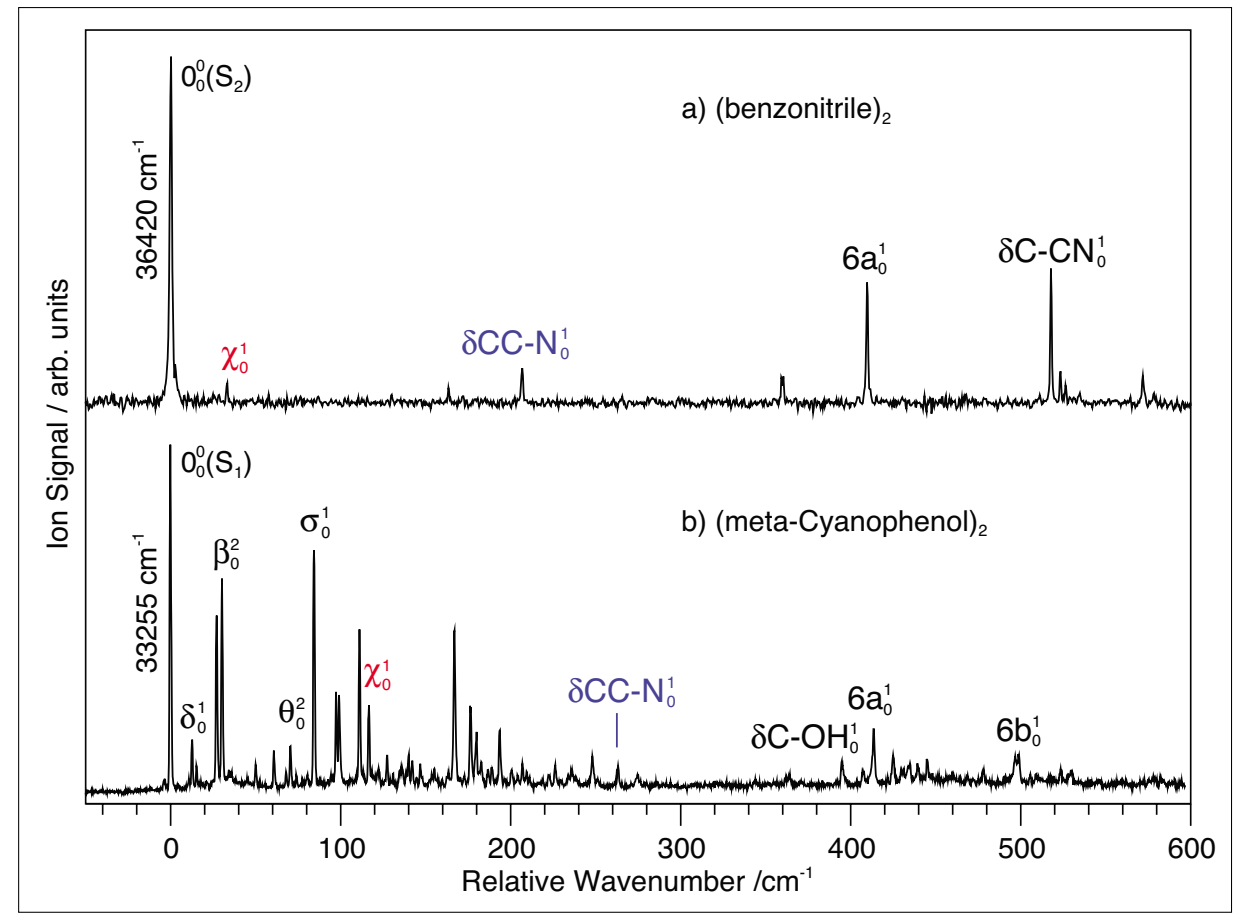

Fig. 2. Two-color resonant two-photon ionization spectra of a) (benzonitrile) ${ }_{2}$ and b) (meta-cyanophenol) $)_{2}$ (b) with assignments. Vibrations appearing in both spectra are color-coded.

Inter- and intramolecular vibrations that involve large motions of the H-bonded atoms and that appear in the spectra of both $(\mathrm{BN})_{2}$ and $(\mathrm{mCP})_{2}$ allow to draw conclusions on H-bond strength. The intermolecular shear vibration $\chi$ and the in-plane cyano-group bend $\delta C C N$ appear in both spectra. The respective fundamental excitations exhibit lower frequencies in $(\mathrm{BN})_{2}$ compared to $(\mathrm{mCP})_{2}: \chi_{0}^{1}$ has an experimental frequency of $33 \mathrm{~cm}^{-1}$ in $(\mathrm{BN})$, and 117 $\mathrm{cm}^{-1}$ in $(\mathrm{mCP})_{2}$; the $\delta \mathrm{CCN}_{0}^{1}$ is at $206 \mathrm{~cm}^{-1}$ in
$(\mathrm{BN})_{2}$ and $263 \mathrm{~cm}^{-1}$ in $(\mathrm{mCP})_{2}$. This shows that the $\mathrm{OH} \cdots \mathrm{N} \equiv \mathrm{C} \mathrm{H}$-bond is much stronger compared to the $\mathrm{CH}^{\cdots} \mathrm{N} \equiv \mathrm{C}$ bond, because more energy is required to deform it. This is confirmed by the SCS-CC2/aVTZ calculated and counterpoise-corrected intermolecular $S_{0}$ binding energies, which are $D_{e}^{C P C}=-57.9 \mathrm{~kJ} / \mathrm{mol}$ for $(\mathrm{mCP})$, and $D_{e}^{C P C}=-23.1 \mathrm{~kJ} / \mathrm{mol}$ for $(\mathrm{BN})_{2}$, with corresponding basis-set superposition errors of $9.5 \mathrm{~kJ} / \mathrm{mol}$ and $4.3 \mathrm{~kJ} / \mathrm{mol}$, respectively. 


\section{Excitonic Splitting and Vibronic Coupling}

Isotopic ${ }^{12} \mathrm{C} /{ }^{13} \mathrm{C}$ substitution at a single atom breaks the inversion symmetry of the dimers and the symmetry descent, which is $C_{2 h} \rightarrow C_{s}$ for $(\mathrm{BN})_{2}$ and $C_{i} \rightarrow C_{1}$ for $(\mathrm{mCP})_{2}$, renders the forbidden transition allowed. We take advantage of the natural abundance of ${ }^{13} \mathrm{C}$. Fig. 3 shows the mass-selective 2C-R2PI spectra of $(\mathrm{BN})_{2}$ and $(\mathrm{mCP})$ in the $0_{0}^{0}$ region, where the top panels display the all- ${ }^{12} \mathrm{C}$ mass channel and the lower panels the ${ }^{13} \mathrm{C}$-trace. In the case of $(\mathrm{BN})_{2}{ }^{-13} \mathrm{C}$ the $S_{0} \rightarrow S_{1} 0_{0}^{0}$ transition appears at $36418.9 \mathrm{~cm}^{-1}$, red-shifted by $1.2 \mathrm{~cm}^{-1}$ relative to $(\mathrm{BN})_{2}$, and with an $S_{1} / S_{2}$ splitting of $\Delta_{\text {obs }}=3.9 \mathrm{~cm}^{-1}$,[13] see Fig. 3(a). The $S_{0} \rightarrow S_{2}$ transition of $(\mathrm{BN})_{2}{ }^{-13} \mathrm{C}$ is blue-shifted by $2.7 \mathrm{~cm}^{-1}$ relative to the $(\mathrm{BN})_{2} S_{2}$ origin, which is due to changes in the zero-point vibrational energy (ZPVE) by isotopic substitution $\Delta_{\text {iso }}$. The observed $S_{1} / S_{2}$ splitting thus comprises an excitonic contribution $\Delta_{\text {exc }}$ and an isotopic contribution $\Delta_{\text {iso }}$. According to degenerate perturbation theory, these combine as: ${ }^{[4,13]}$

$$
\Delta_{o b s}=\sqrt{\Delta_{e x c}^{2}+\Delta_{i s o}^{2}}
$$

From monomer 2C-R2PI spectra of ${ }^{12} \mathrm{C}-\mathrm{BN}$ and ${ }^{13} \mathrm{C}-\mathrm{BN}$ we have determined the purely isotopic contribution to be $\Delta_{\text {iso }}=3.3 \mathrm{~cm}^{-1}$, leading to an excitonic splitting of $\Delta_{\text {exc }}=2.1 \mathrm{~cm}^{-1}$. ${ }^{[13]}$

In the case of $(\mathrm{mCP})_{2}$ the forbidden $\mathrm{S}_{2} \mathrm{O}_{0}^{0}$ band appears very weakly in the $(\mathrm{mCP})-{ }^{13} \mathrm{C}$ spectrum at $8.0 \mathrm{~cm}^{-1}$ above the $S_{1}$ origin, see Fig. 3(b). The $\mathrm{S}_{1} 0_{0}^{0}$ band of $(\mathrm{mCP}){ }_{2}{ }^{13} \mathrm{C}$ is hardly shifted compared to that of $(\mathrm{mCP})$, indicating smaller changes in ZPVE. [9] The $S_{0} \rightarrow S_{1}$ spectra of mCP and $\mathrm{mCP}-{ }^{13} \mathrm{C}$ were used to determine the isotopic contribution $\Delta_{\text {iso }}=3.3 \mathrm{~cm}^{-1}$ as well. Employing Eqn. (2) with $\Delta_{\text {obs }}=8.0 \mathrm{~cm}^{-1}$ yields an excitonic splitting of $\Delta_{\text {exc }}=7.3$ $\mathrm{cm}^{-1}$.

The vertical excitation energies calculated at the optimized SCS-CC2/aVTZ ground-state structures predict purely electronic splittings of $\Delta_{\text {calc }}=10 \mathrm{~cm}^{-1}$ for $(\mathrm{BN})_{2}$ and $\Delta_{\text {calc }}=179 \mathrm{~cm}^{-1}$ for $(\mathrm{mCP})_{2}$. However, these splittings do not take the redistribution of the electronic TDM into vibronic TDMs into account, and can therefore not be directly compared to the determined excitonic splittings $\Delta_{\text {exc }}{ }^{[3-7,9,10]}$ To calculate the vibronic quenching factor $\Gamma=\Pi_{i} \exp \left(-S_{i}\right)=\exp \left(-\Sigma_{i} S_{i}\right)$, we employed the Huang-Rhys factors $S_{i}=l_{i} / 2 \mu_{i} \hbar \omega_{i}^{3}$, where $i$ numbers the totally-symmetric intramolecular vibrations of the $\mathrm{BN}$ or $\mathrm{mCP}$ monomer. ${ }^{[3-6]}$ The experimental $S_{i}$ yielded $\Gamma_{\mathrm{exp}}=0.213$ for $(\mathrm{BN})_{2}$ and $\Gamma_{\exp }=0.043$ for $(\mathrm{mCP})_{2} \cdot{ }^{[9,13]}$ The vibronic coupling hence

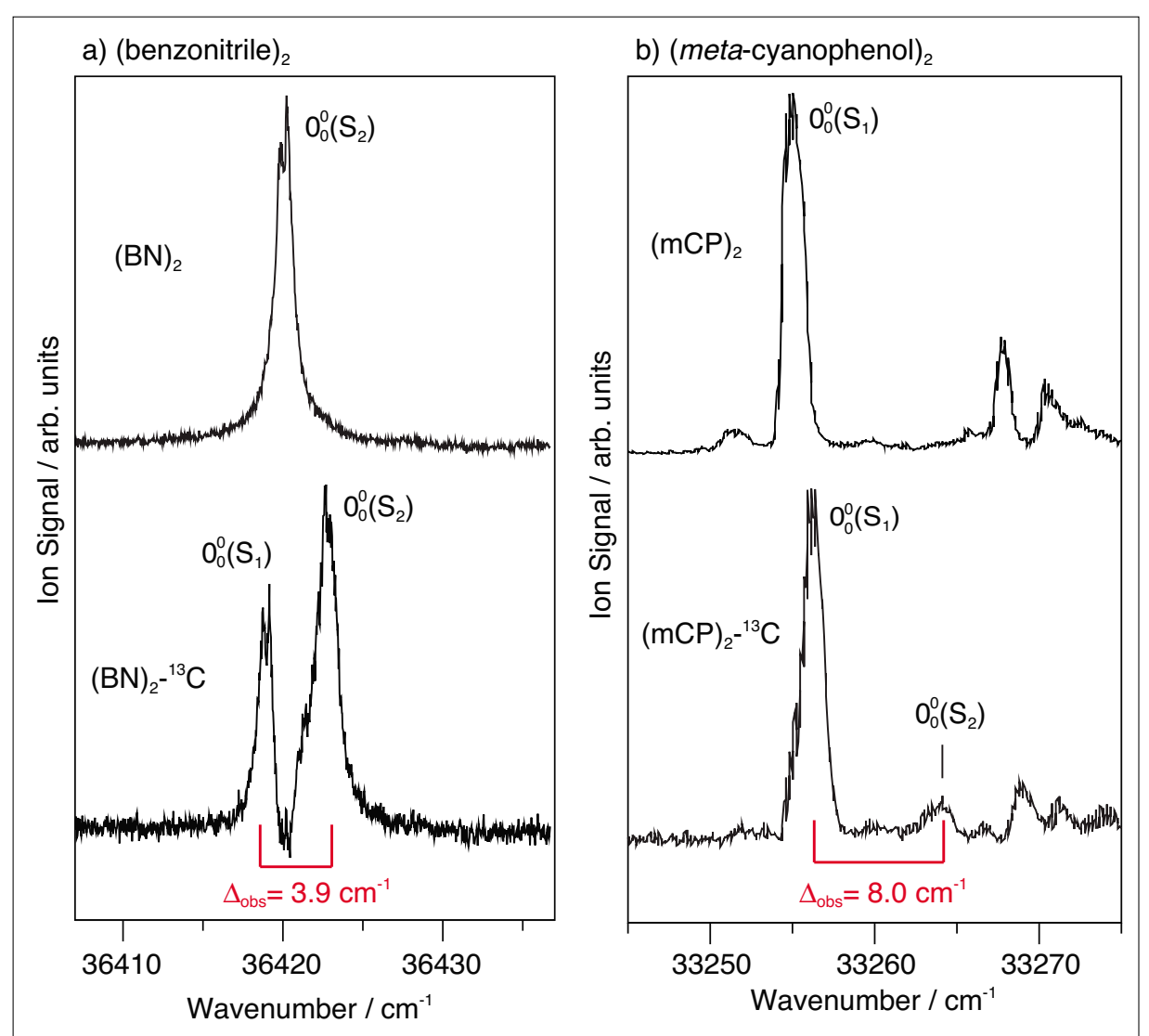

Fig. 3. 2C-R2PI spectra in the origin region of a) (benzonitrile) ${ }_{2}$ and b) (meta-cyanophenol) ${ }_{2}$ in the ${ }^{12} \mathrm{C}$-mass channel (top) and the ${ }^{13} \mathrm{C}$-mass channel (bottom) with observed $\mathrm{S}_{1} / \mathrm{S}_{2}$ splitting in red.

quenches the Davydov splittings by factors of $\Gamma^{-1}=5$ and 23 , respectively, leading to vibronic $S_{l} / S_{2}$ splittings of $\Delta_{\text {vibron }}=2.1 \mathrm{~cm}^{-1}$ for $(\mathrm{BN})_{2}$ and $\Delta_{\text {vibron }}=7.7 \mathrm{~cm}^{-1}$ for $(\mathrm{mCP})_{2}$. This is in excellent agreement with the excitonic splittings determined above; all the splittings are summarized in Table 1. We note that these splittings arise from properties of the monomers: The excitonic splitting results due to coupling of the monomer TDMs and the vibronic quenching is largely dependent on intramolecular monomer vibrations $Q_{i}$ and Huang-Rhys factors $S_{i}$. This implies that the H-bond strength of the dimer does not affect the excitonic splitting or vibronic quenching.

Table.1. Excitonic $S_{1} / S_{2}$ splitting $\left(\Delta_{\text {exc }}\right)$, Davydov splitting $\left(\Delta_{\text {cald }}\right)$, quenching factor $(\Gamma)$, resulting vibronic splitting $\left(\Delta_{\text {vibron }}=\Gamma \cdot \Delta_{\text {calc }}\right)$ and site-shift $(\delta v)$.

\begin{tabular}{|c|c|c|}
\hline & (benzonitrile) & $\begin{array}{c}\text { (m-cyano- } \\
\text { phenol) }\end{array}$ \\
\hline$\Delta_{\text {exc }}$ & $2.0 \mathrm{~cm}^{-1}$ & $7.3 \mathrm{~cm}^{-1}$ \\
\hline$\Delta_{\text {calc }}$ & $10 \mathrm{~cm}^{-1}$ & $179 \mathrm{~cm}^{-1}$ \\
\hline$\Gamma$ & 0.213 & 0.043 \\
\hline$\Delta_{\text {vibron }}$ & $2.1 \mathrm{~cm}^{-1}$ & $7.7 \mathrm{~cm}^{-1}$ \\
\hline$\delta v$ & $-92 \mathrm{~cm}^{-1}$ & $-1094 \mathrm{~cm}^{-1}$ \\
\hline
\end{tabular}

\section{Spectral Site-Shifts}

The intermolecular interactions that occur upon dimerization act on the constituent monomers and result in changes of the monomer geometry as well as energy (the so-called deformation energy). The geometry deformation of the monomer leads to a change of its electronic structure. This change is different in the $S_{0}$ and $S_{1}$ states, leading to a shift of the adiabatic $S_{0} \rightarrow S_{1}$ absorption frequency. Thus the dimerization causes an electronic spectral site-shift. The site-shift can be evaluated from the average of spectral shifts of the dimer $S_{1} / S_{2}$ $0_{0}^{0}$ bands relative to the monomer $S_{0} \rightarrow S_{1}$ $0_{0}^{0}$ band, see Fig. 4 .

Fig. 4 shows that the excitonic splitting discussed above is relatively small and does not depend on the H-bond strength. In contrast, the site-shift leads to much larger spectral effects and largely depends on the $\mathrm{H}$-bond strength. The $\mathrm{BN} S_{0} \rightarrow S_{1} 0_{0}^{0}$ band is observed at $36513 \mathrm{~cm}^{-1}[18]$ and the average of the dimer $S_{0} \rightarrow S_{1} / S_{2}$ transitions is $36420 \mathrm{~cm}^{-1}$. ${ }^{[13]}$ This result corresponds to a small site-shift of $\Delta_{\text {site }}=-92 \mathrm{~cm}^{-1}$, which is $5 \%$ of the binding energy. The $\mathrm{mCP}$ monomer origin lies at $34354 \mathrm{~cm}^{-1}$ while the average of the dimer $S_{0} \rightarrow S_{1} / S_{2}$ origins is at $33260 \mathrm{~cm}^{-1}$, resulting in a much larger site-shift of $\Delta_{\text {site }}=-1094 \mathrm{~cm}^{-1}$. Here, the site-stabilization is $23 \%$ of the dimer binding energy. This shows that the $\mathrm{CH} \cdots \mathrm{N} \equiv \mathrm{C}$ 


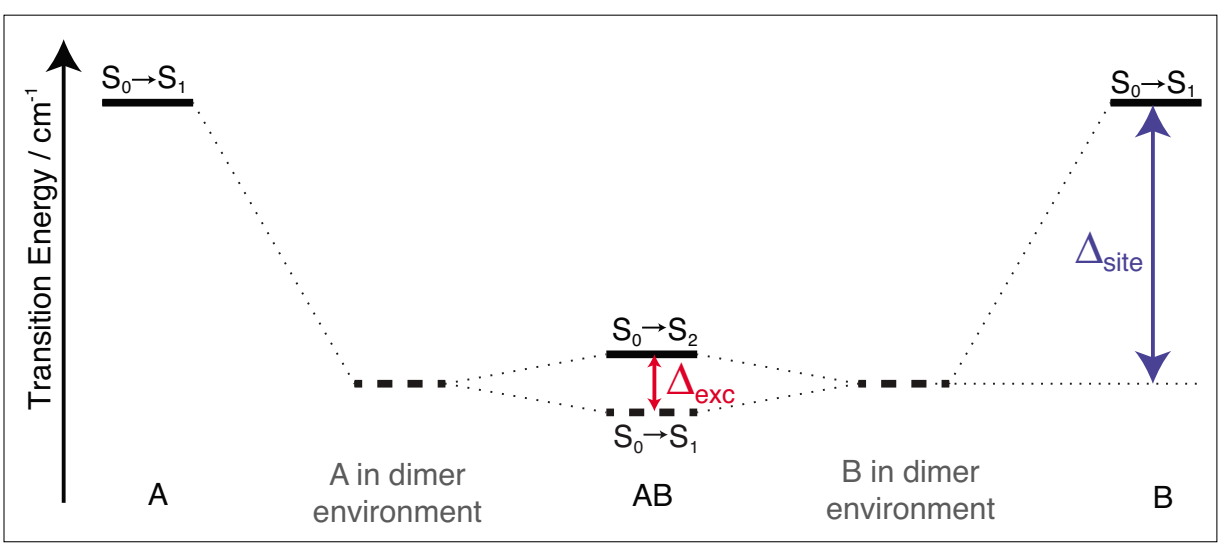

Fig. 4. Schematic representation of site- and excitonic splitting in a homodimer, where the $S_{0} \rightarrow S_{1}$ transition is forbidden. Experimentally accessible values are marked in full lines, while calculated or indirectly determined transitions are dashed.

bonds have a much smaller influence than the $\mathrm{OH} \cdots \mathrm{N} \equiv \mathrm{C}$ bonds, as the stabilization is much larger in $(\mathrm{mCP})_{2}$.

\section{Conclusions}

Jet-cooled two-color resonant twophoton ionization spectra of (benzonitrile), and (meta-cyanophenol) ${ }_{2}$ and their ${ }^{13} \mathrm{C}$-isotopomers revealed excitonic splittings of $\Delta_{\text {exc }}=2.1 \mathrm{~cm}^{-1}$ and $\Delta_{\text {exc }}=7.3 \mathrm{~cm}^{-1}$, respectively. The vibronic quenching factor derived from monomer fluorescence spectra was much smaller for $(\mathrm{mCP})_{2}$ $(\Gamma=0.043)$ than for $(\mathrm{BN}),(\Gamma=0.213)$, since $2 \mathrm{C}$-R2PI spectrum of $\mathrm{mCP}$ exhibits many more vibronic bands than BN. This leads to a much stronger quenching of the electronic exciton splitting by a factor $\Gamma^{-1}=23$, while in $(\mathrm{BN})_{2} \Gamma^{-1}=5$. The hy- larger than the excitonic splittings and are heavily influenced by the hydrogen bond strengths. Thus the H-bonds have large effects on the spectral shifts, but little or no influence on the exciton splitting.

Received: January 21, 2016

[1] F. C. Spano, Аnпu. Rev. Phys. Chem. 2006, 57, 4563.

[2] P. Ottiger, S. Leutwyler, H. Köppel, J. Chem. Phys. 2009, 131, 204308.

[3] P. Ottiger, S. Leutwyler, H. Köppel, J. Chem. Phys. 2012, 136, 174308.

[4] P. Ottiger, S. Leutwyler, J. Chem. Phys. 2012, $137,204303$.

[5] S. Kopec, P. Ottiger, S. Leutwyler, H. Köppel, J. Chem. Phys. 2015, 142, 084308.

[6] P. Ottiger, S. Leutwyler, H. Köppel, Chem. Sci. 2015, 6, 6059 .

[7] T. Förster, in 'Modern Quantum Chemistry', Ed. O. Sinanoglu, Academic, New York and London, 1965, Chap. IIIB, p. 93.

[8] A. Witkowski, W. Moffitt, J. Chem. Phys. 1960, 33,872 .

[9] F. A. Balmer, P. Ottiger, S. Kopec, H. Köppel, S. Leutwyler, to be submitted.

[10] S. Kopec, P. Ottiger, S. Leutwyler, H. Köppel, J. Chem. Phys. 2012, 137, 184312.

[11] R. L. Fulton, M. Gouterman, J. Chem. Phys. 1961, 35, 1059.

[12] R. L. Fulton, M. Gouterman, J. Chem. Phys. 1964, 41, 2280.

[13] F. A. Balmer, P. Ottiger, S. Leutwyler, J. Phys. Chem. A 2014, 118, 11253.

[14] T. Kobayashi, K. Honma, O. Kajimoto, S. Tsuchiya, J. Chem. Phys. 1987, 86, 1111.

[15] N. Seurre, K. Le Barbu-Débus, F. Lahmani, A. Zehnacker-Rentien, J. Sepiol, Chem. Phys. 2003, 295, 21

[16] E. B. Wilson, Phys. Rev. 1934, 45, 706

[17] A. Müller, F. Talbot, S. Leutwyler, J. Am. Chem. Soc. 2002, 124, 14486.

[18] P. Imhof, D. Krügler, R. Brause, K. Kleinermanns, J. Chem. Phys. 2004, 121, 2598. 